\title{
First-Line Treatment of Widely Metastatic BRAF-Mutated Salivary Duct Carcinoma With Combined BRAF and MEK Inhibition
}

\author{
Victor T.G. Lin, MD, PhDa; Lisle M. Nabell, MDª, ; Sharon A. Spencer, MD ${ }^{\mathrm{b}, \mathrm{c}}$; William R. Carroll, MD ${ }^{\mathrm{b}, \mathrm{d}}$; \\ Shuko Harada, MD ${ }^{\mathrm{b}, \mathrm{e}}$; and Eddy S. Yang, MD, PhD ${ }^{\mathrm{b}, \mathrm{c}}$
}

\section{Abstract}

Salivary duct carcinoma (SDC) is a rare and aggressive malignancy for which limited data exist to guide treatment decisions. With the advent of advanced molecular testing and tumor genomic profiling, clinicians now have the ability to identify potential therapeutic targets in difficult-to-treat cancers such as SDC. This report presents a male patient with widely metastatic SDC found on targeted next-generation sequencing to have a $B R A F$ p.V600E mutation. He experienced a prolonged and robust response to first-line systemic chemotherapy with dabrafenib and trametinib. During his response interval, new data emerged to justify subsequent treatment with both an immune checkpoint inhibitor and androgen blockade after his disease progressed. To our knowledge, this is the first report of frontline BRAF-directed therapy eliciting a response in metastatic SDC.

J Natl Compr Canc Netw 2018;16(10):1166-1170 doi: 10.6004/jnccn.2018.7056

Salivary gland cancers are a heterogeneous collection of malignancies defined primarily by different histologies, which include adenoid cystic carcinoma (ACC), mucoepidermoid carcinoma, and salivary duct carcinoma (SDC). In particular, SDC is a neoplasm known for both its poor prognosis and its rarity. Because of these characteristics, data are currently insufficient to confidently recommend a standard of care for these tumors.

Recently, clinical oncologists have had increasing access to tumor genomic profiling, which allows for identification of mutations, gene fusions, and copy number variants that may predict response to specific targeted therapies. This approach has been of particular interest in so-called difficult-to-treat cancers, which include rare malignancies such as SDC, that have a very limited evidence base to guide treatment decisions. This report presents a patient with a widely metastatic SDC harboring a BRAF p.V600E mutation that was treated with a precision oncology approach developed in conjunction with the University of Alabama at Bir-

aDivision of Hematology and Oncology, Department of Medicine, University of Alabama at Birmingham; ${ }^{b}$ University of Alabama at

Birmingham Comprehensive Cancer Center; and Departments of 'Radiation

Oncology, 'Otolaryngology, and ePathology, University of Alabama at

Birmingham, Birmingham, Alabama.

Submitted February 22, 2018; accepted for publication June 29, 2018. mingham (UAB) Molecular Tumor Board (MTB). ${ }^{1}$ To our knowledge, this is the first reported use of combined BRAF and MEK inhibition for the treatment of metastatic BRAF-mutated SDC in the frontline setting.

\section{Case Report}

A 54-year-old white male presented initially with 6 weeks of swelling in the left cheek and neck that failed to improve with clindamycin. CT imaging showed multiple intraparotid nodules and cervical lymphadenopathy. Ultrasound-guided core biopsies were positive for indeterminate carcinoma. Staging by PET/CT showed involvement of the left cervical nodes with no definite distant metastases. He was subsequently referred to UAB, where he underwent direct laryngoscopy with biopsy, followed by a left modified radical neck dissection and left total parotidectomy. Pathology from his surgical resection revealed a high-grade, locally advanced (pT4aN2b,cM0) SDC with multiple high-risk features, including a posi-

The authors have disclosed that they have no financial interests, arrangements, affiliations, or commercial interests with the manufacturers of any products discussed in this article or their competitors.

Correspondence: Eddy S. Yang, MD, PhD, Department of Radiation

Oncology, University of Alabama at Birmingham, 1700 6th Avenue S,

HSROC Suite 2232N, Birmingham, AL 35249. Email: shyang@uabmc.edu 
tive margin of resection, extracapsular extension, and tumor involvement of 31 of 40 resected lymph nodes. He was enrolled in a clinical trial comparing adjuvant chemoradiation versus radiation therapy (RT) alone (RTOG 1008), and was randomly assigned to treatment with RT alone.

Three months after completing adjuvant RT, he was found to have widespread osseous metastases with involvement of the intrathoracic lymph nodes. After HER2 amplification was ruled out by immunohistochemistry and in situ hybridization, tumor tissue was sent to Genomics and Pathology Services at Washington University in St. Louis for targeted next-generation sequencing (NGS; gene list shown in supplemental eAppendix 1, available with this article at JNCCN. org), and a BRAF p.V600E mutation was identified. After discussion by the UAB MTB,${ }^{1}$ he was started on targeted therapy with the BRAF inhibitor dabrafenib and the MEK inhibitor trametinib, in addition to zoledronic acid for his bony metastases (Figure 1). Dabrafenib and trametinib were held approximately a month after initiation due to grade 4 palmar-plantar erythrodysesthesia, and were subsequently restarted at reduced doses, which he tolerated well. Restaging PET/CT was performed after 5 months of treatment and showed marked improvement in his diffuse osseous metastases (Figure 2A, B). He continued on therapy, and restaging PET/CT after 9 total months of treatment showed essentially stable disease with the exception of a new hypermetabolic lesion in the right ischium, concerning for new metastasis (Figure 2C). He continued on the same regimen due to ineligibil-

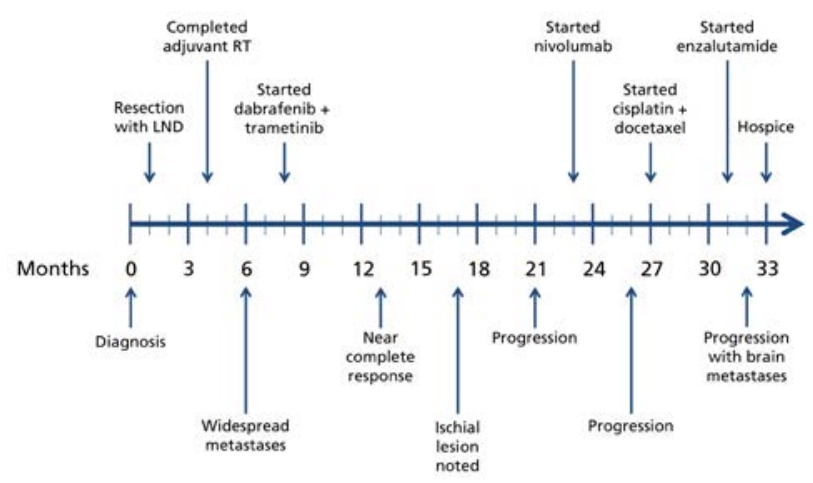

Figure 1. Timeline summary of clinical course. Lines of treatment are listed above the timeline and changes in disease status by restaging imaging are shown below it. Dose reductions, holds, and discontinuations, and palliative radiotherapies are not shown for the sake of simplicity.

Abbreviations: LND, lymph node dissection; RT, radiation therapy. ity for phase I clinical trials and limited alternatives in the setting of mostly stable disease. Restaging PET/ CT performed after 13 total months of treatment revealed multiple new bony metastases (Figure 2D), at which point dabrafenib and trametinib were re-escalated to full doses without any of the prior toxicities. Shortly thereafter, he underwent a surgical biopsy of a posterior sacral metastasis, which was submitted to PierianDx for repeat targeted NGS (gene list shown in supplemental eAppendix 2) and did not find any new actionable mutations. With clinical evidence of progression, including a rising alkaline phosphatase that had previously normalized with treatment, dab-
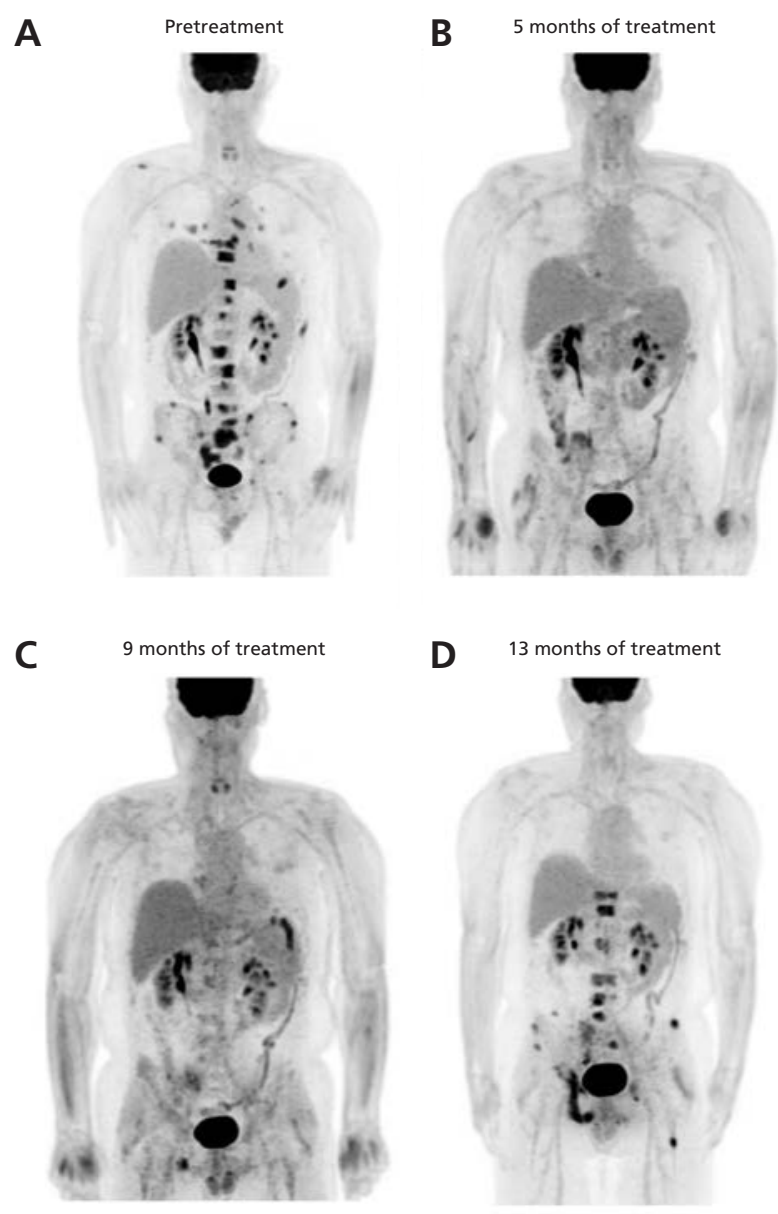

Figure 2. Marked response to dabrafenib and trametinib demonstrated by restaging imaging. PET/CT scans performed before and after initiation of treatment with dabrafenib and trametinib show a robust response to treatment. Pretreatment and posttreatment scans were performed at $6,13,17$, and 21 months after initial diagnosis ( $A-D$, respectively). Most strikingly, there is complete resolution of the vertebral lesions. The new right ischial lesion noted in (C) portended the progression of disease through treatment that was demonstrated 3 months later. 
Lin et al

rafenib and trametinib were discontinued and he received palliative RT to the hip.

At this time, a petition to the manufacturer for use of nivolumab as part of an expanded access program was approved, and he was started on immune checkpoint blockade after completion of RT. For additional information, peripheral blood was sent for analysis of circulating tumor DNA (ctDNA) by Guardant360 (Guardant Health, Inc.), revealing the known BRAF mutation with a variant allele frequency (VAF) of $28 \%$ and a KRAS p.Q61K mutation with a low VAF of $0.2 \%$, as well as amplifications of MET, EGFR, and CDK6 of unclear clinical significance. This new information was brought again to the UAB MTB for discussion. Tyrosine kinase inhibitors (TKIs) were considered but not recommended due to the downstream activating mutation of BRAF. CDK inhibitors were also discussed, but not pursued. He remained on nivolumab for 3 months before discontinuation for severe fatigue unrelated to iatrogenic hypothyroidism or adrenal insufficiency.

The patient was subsequently started on conventional chemotherapy with cisplatin and docetaxel. Over the next 3 months, he completed 5 cycles of cisplatin and docetaxel and 1 cycle of cisplatin alone, with docetaxel being dose-reduced and eventually discontinued due to progressively worsening neuropathy. Although restaging by PET/CT showed a partial response to cisplatin and docetaxel, he was unable to continue these agents due to the aforementioned toxicity. During this time, tumor tissue from a prior biopsy was tested for androgen receptor (AR) by immunohistochemistry, with expression noted in $60 \%$ of cells, and was therefore approved for off-label use of enzalutamide. He continued on this for approximately 1 month, at which point he was evaluated for intractable headaches and found to have diffuse brain metastases. He subsequently underwent palliative wholebrain RT before being enrolled in hospice.

\section{Discussion}

To date, sparse evidence supports the use of conventional chemotherapy in the treatment of salivary gland tumors. Histologic subtypes of salivary gland cancers can be segregated by the expression of specific molecular markers and may accordingly respond differently to conventional and targeted chemotherapeutic agents. ${ }^{2}$ For instance, ACC tends to express the receptor tyrosine kinase (RTK) c-Kit and is known to be poorly responsive to not only conventional chemotherapy ${ }^{3}$ but also TKIs with activity against c-Kit, such as imatinib ${ }^{4}$ and dasatinib. ${ }^{5}$ In contrast, a recent retrospective review of patients with SDC suggested a benefit to treatment with combined carboplatin and paclitaxel. ${ }^{6}$ Notably, this cohort of 18 patients was established from a retrospective review of $>8$ years of clinical records, which exemplifies the difficulty of performing robust prospective clinical trials in this type of cancer. A larger retrospective evaluation of 495 patients with SDC listed in the National Cancer Database indicated that surgical resection was superior to all other modalities, with insufficient data available to properly evaluate the role of conventional or targeted chemotherapies. ${ }^{\text {? }}$

Interestingly, SDC histologically resembles invasive ductal carcinoma of the breast, ${ }^{8}$ but its genomic profile is most similar to that of apocrine breast cancer. ${ }^{9}$ Therefore, it only rarely expresses estrogen or progesterone receptors, but has been associated with expression of HER2 and AR, both of which might be markers for response to targeted therapies. ${ }^{2}$ Retrospective studies have suggested that targeting HER2 with trastuzumab may be of benefit in HER2-expressing SDC,${ }^{10}$ and interim results from a prospective phase II trial of docetaxel plus trastuzumab were encouraging. ${ }^{11}$ Furthermore, a recent case report suggested that trastuzumab emtansine (T-DM1) may have utility after trastuzumab failure. ${ }^{12}$ Although AR expression in SDC has long been reported, ${ }^{13}$ data supporting the use of androgen deprivation therapy (ADT) are limited to case series.,14 Given the paucity of SDCs, currently active clinical trials are open to all AR-positive salivary gland cancers. Perhaps the most intriguing is EORTC 1206 (ClinicalTrials.gov identifier: NCT01969578), which is designed to compare conventional chemotherapy versus standard ADT consisting of the first-generation AR antagonist bicalutamide and triptorelin, a gonadotropin-releasing hormone agonist. Other ongoing noncomparative trials seek to evaluate the efficacy of second-line ADTs, such as the second-generation AR antagonist enzalutamide (NCT02749903) and the CYP17 inhibitor abiraterone (NCT02867852).

In our patient, tumor genomic profiling identified a BRAF p.V600E mutation, which is commonly thought to be a true oncogenic driver targetable by combined BRAF and MEK inhibition. Combination 
of dabrafenib with trametinib was first approved for use in metastatic melanoma harboring V600 mutations. ${ }^{15}$ A subsequent trial demonstrated this combination could also be efficacious in the salvage setting for BRAF V600E metastatic non-small cell lung cancer (NSCLC). ${ }^{16}$ BRAF V600E mutations are characteristic of classical hairy cell leukemia (HCL), and treatment with the BRAF inhibitor vemurafenib confers some benefit. ${ }^{17}$ Combination with a MEK inhibitor in HCL has not yet been evaluated in a clinical trial, but preclinical data suggest that this may be more effective than using a BRAF inhibitor alone. ${ }^{18}$ Most recently, the FDA also approved the use of dabrafenib and trametinib in the treatment of anaplastic thyroid carcinoma harboring a BRAF V600E mutation, which represents up to $50 \%$ of these tumors overall. ${ }^{19}$ Responses to targeted therapies have generally been better with fewer prior lines of treatment, and recent trials have shown that BRAF-directed therapy may be used upfront as adjuvant therapy in resected stage III melanoma ${ }^{20}$ and as first-line therapy in metastatic NSCLC. ${ }^{21}$ Although BRAF V600 mutations are much less common in other malignancies, cases demonstrating the efficacy of combined BRAF and MEK inhibition have been reported in a number of other diverse cancers, including high-grade colorectal neuroendocrine tumors, ${ }^{22}$ papillary thyroid carcinoma, ${ }^{23}$ and endometrial cancer. ${ }^{24}$

To our knowledge, this is the first report of a patient with BRAF-mutated SDC treated with combined BRAF and MEK inhibition in the first-line setting for metastatic disease. A prior case report described the use of vemurafenib alone after disease progression occurred during treatment with combination 5-fluorouracil and cisplatin in a patient with BRAF V600E-mutated parotid cystadenocarcinoma. ${ }^{25} \mathrm{An}$ other case reported on a series of patients with SDC whose tumors were analyzed using targeted mutational analysis, and described in detail one patient who harbored a BRAF V600E mutation. ${ }^{26}$ That patient experienced disease progression after definitive concurrent chemoradiotherapy with weekly carboplatin and paclitaxel, and again after salvage chemotherapy with cyclophosphamide and doxorubicin. He was treated with an unidentified MEK inhibitor on study before disease progression, after which he was enrolled in a phase I trial of combined dabrafenib and trametinib. Like these other cases, our patient was treated with targeted therapy directed against BRAF V600E. How- ever, we elected to undertake this approach in the first-line setting due to his widely metastatic disease, and observed a similarly robust response to treatment resulting in the radiographic resolution of his metastatic lesions within 6 months that was fairly durable, lasting $>1$ year.

After our patient experienced disease progression on dabrafenib and trametinib, we performed sequencing of both a sacral metastasis and ctDNA. In addition to the known BRAF V600E mutation, analysis of ctDNA showed amplifications in CDK6 and the RTKs MET and EGFR, as well as a very low frequency KRAS Q61K-activating mutation. Any of these alterations may have plausibly contributed to treatment resistance in this case. The amplification of CDK6, which is a phosphorylation target and effector of the mitogen-activated protein kinase (MAPK) pathway, likely leads to BRAF inhibitor resistance similar to more commonly seen alterations in CDK4 and cyclin D1. ${ }^{27}$ Prior studies have demonstrated that the resistance of BRAF V600 mutants to BRAF inhibitors may arise from upregulation of RTKs or activating mutations of NRAS or KRAS upstream of BRAF and MEK, but that MAPK reactivation through these routes conferred sensitivity to MEK inhibition. ${ }^{28,29}$ KRAS mutants are also less sensitive than BRAF V600 mutants to some MEK inhibitors via a CRAF-dependent mechanism; however, trametinib is more effective than earlier-generation MEK inhibitors at suppressing downstream ERK activation in KRAS mutants. ${ }^{29}$ Although all of these mechanisms are known to explain resistance to dabrafenib, only the CDK6 amplification represents a bypass occurring downstream of BRAF and MEK. Given the limitations of targeted sequencing, unidentified alterations in untested genes, as well as epigenetic and posttranscriptional and posttranslational changes, may have also contributed to treatment resistance.

Importantly, our patient's prolonged response allowed for the emergence of other potential approaches. Shortly after his disease progressed on dabrafenib and trametinib, preliminary results of the salivary arm of the KEYNOTE-028 trial of pembrolizumab were reported and suggested a possible role for immunotherapy. ${ }^{30}$ These data provided a rationale to petition for the use of nivolumab, which was granted. Unfortunately, our patient was unable to tolerate this treatment due to severe fatigue, and it was discontinued. Chemotherapy with cisplatin and docetaxel was 
Lin et al

subsequently initiated. After becoming intolerant to these conventional agents, he was briefly started on ADT with enzalutamide, but was unfortunately found to have brain metastases before a response could be accurately assessed.

\section{Conclusions}

Standard of care for SDC remains elusive, especially in light of potentially actionable mutations associated with it. The most effective combinations and sequencing of therapy are open questions in the treatment of this disease. Because of the rarity of this cancer, advances will likely be incumbent on large-scale precision oncology trials, such as NCIMATCH and ASCO's TAPUR, and on the approval of tumor-agnostic molecularly targeted approaches paired with tumor genomic profiling. Although BRAF V600E mutations are thought to be uncommon in SDC, we provide evidence that combined BRAF and MEK inhibition is a reasonable first-line option for metastatic disease.

\section{References}

1. Harada S, Arend R, Dai Q, et al. Implementation and utilization of the molecular tumor board to guide precision medicine. Oncotarget 2017;8:57845-57854

2. Alfieri S, Granata R, Bergamini C, et al. Systemic therapy in metastatic salivary gland carcinomas: a pathology-driven paradigm? Oral Oncol 2017;66:58-63.

3. Laurie SA, Ho AL, Fury MG, et al. Systemic therapy in the management of metastatic or locally recurrent adenoid cystic carcinoma of the salivary glands: a systematic review. Lancet Oncol 2011;12:815-824.

4. Pfeffer MR, Talmi $Y$, Catane R, et al. A phase II study of imatinib for advanced adenoid cystic carcinoma of head and neck salivary glands. Oral Oncol 2007;43:33-36.

5. Wong SJ, Karrison T, Hayes DN, et al. Phase II trial of dasatinib for recurrent or metastatic c-KIT expressing adenoid cystic carcinoma and for nonadenoid cystic malignant salivary tumors. Ann Oncol 2016;27:318-323.

6. Nakano K, Sato Y, Sasaki T, et al. Combination chemotherapy of carboplatin and paclitaxel for advanced/metastatic salivary gland carcinoma patients: differences in responses by different pathological diagnoses. Acta Otolaryngol 2016;136:948-951.

7. Osborn V, Givi B, Lee A, et al. Characterization, treatment and outcomes of salivary ductal carcinoma using the National Cancer Database. Oral Oncol 2017;71:41-46.

8. Jaspers HC, Verbist BM, Schoffelen R, et al. Androgen receptor-positive salivary duct carcinoma: a disease entity with promising new treatment options. J Clin Oncol 2011;29:e473-476.

9. Dalin MG, Desrichard A, Katabi N, et al. Comprehensive molecular characterization of salivary duct carcinoma reveals actionable targets and similarity to apocrine breast cancer. Clin Cancer Res 2016;22:4623-4633.

10. Limaye SA, Posner MR, Krane JF, et al. Trastuzumab for the treatment of salivary duct carcinoma. Oncologist 2013;18:294-300.

11. Takahashi $H$, Masubuchi $T$, Fushimi C, et al. Trastuzumab and docetaxel for HER2-positive unresectable salivary gland carcinoma: updated results of a phase II trial [abstract]. Presented at the 2016 AHNS Annual Meeting; July 16-20, 2016; Seattle, Washington.

12. van Boxtel W, Boon E, Weijs WL, et al. Combination of docetaxel, trastuzumab and pertuzumab or treatment with trastuzumab-emtansine for metastatic salivary duct carcinoma. Oral Oncol 2017;72:198-200.

13. Fan CY, Wang J, Barnes EL. Expression of androgen receptor and prostatic specific markers in salivary duct carcinoma: an immunohistochemical analysis of 13 cases and review of the literature. Am J Surg Pathol 2000;24:579-586.

14. Yamamoto N, Minami S, Fujii M. Clinicopathologic study of salivary duct carcinoma and the efficacy of androgen deprivation therapy. Am J Otolaryngol 2014;35:731-735.
15. Flaherty KT, Infante JR, Daud $A$, et al. Combined BRAF and MEK inhibition in melanoma with BRAF V600 mutations. N Engl J Med 2012;367:1694-1703.

16. Planchard D, Besse B, Groen HJ, et al. Dabrafenib plus trametinib in patients with previously treated BRAF(V600E)-mutant metastatic non-smal cell lung cancer: an open-label, multicentre phase 2 trial. Lancet Oncol 2016;17:984-993.

17. Tiacci E, Park JH, De Carolis L, et al. Targeting mutant BRAF in relapsed or refractory hairy-cell leukemia. N Engl J Med 2015;373:1733-1747.

18. Pettirossi V, Santi $A$, Imperi $E$, et al. BRAF inhibitors reverse the unique molecular signature and phenotype of hairy cell leukemia and exert potent antileukemic activity. Blood 2015;125:1207-1216.

19. Subbiah V, Kreitman RJ, Wainberg ZA, et al. Dabrafenib and trametinib treatment in patients with locally advanced or metastatic BRAF V600mutant anaplastic thyroid cancer. J Clin Oncol 2018;36:7-13.

20. Long GV, Hauschild A, Santinami M, et al. Adjuvant dabrafenib plus trametinib in stage III BRAF-mutated melanoma. N Engl J Med 2017;377:1813-1823.

21. Planchard D, Smit EF, Groen HJ, et al. Dabrafenib plus trametinib in patient with previously untreated BRAFV600E-mutant metastatic non-small-cell lung cancer: an open-label, phase 2 trial. Lancet Oncol 2017;18:1307-1316.

22. Klempner SJ, Gershenhorn B, Tran P, et al. BRAFV600E mutations in high-grade colorectal neuroendocrine tumors may predict responsiveness to BRAF-MEK combination therapy. Cancer Discov 2016;6:594-600.

23. White PS, Pudusseri A, Lee SL, Eton O. Intermittent dosing of dabrafenib and trametinib in metastatic BRAFV600E mutated papillary thyroid cancer: two case reports. Thyroid 2017;27:1201-1205.

24. Moschetta M, Mak G, Hauser J, et al. Dabrafenib and trametinib activity in a patient with BRAF V600E mutated and microsatellite instability high (MSI-H) metastatic endometrial cancer. Exp Hematol Oncol 2017;6:1.

25. Boyrie S, Fauquet I, Rives M, et al. Cystadenocarcinoma of the parotid: case report of a BRAF inhibitor treatment. Springerplus 2013;2:679.

26. Nardi V, Sadow PM, Juric D, et al. Detection of novel actionable genetic changes in salivary duct carcinoma helps direct patient treatment. Clin Cancer Res 2013;19:480-490.

27. Smalley KS, Lioni M, Dalla Palma M, et al. Increased cyclin D1 expression can mediate BRAF inhibitor resistance in BRAF V600E-mutated melanomas. Mol Cancer Ther 2008;7:2876-2883.

28. Nazarian R, Shi $H$, Wang $Q$, et al. Melanomas acquire resistance to B-RAF(V600E) inhibition by RTK or N-RAS upregulation. Nature 2010;468:973-977.

29. Lito P, Saborowski A, Yue J, et al. Disruption of CRAF-mediated MEK activation is required for effective MEK inhibition in KRAS mutant tumors. Cancer Cell 2014;25:697-710.

30. Cohen RB, Delord JP, Doi T, et al. Preliminary results for the advanced salivary gland carcinoma cohort of the phase $1 \mathrm{~b}$ KEYNOTE-028 study of pembrolizumab [abstract]. J Clin Oncol 2016;34(Suppl):Abstract 6017. 